\title{
Engraftment of donor mesenchymal stem cells in chimeric BXSB includes vascular endothelial cells and hepatocytes
}

This article was published in the following Dove Press journal:

Stem Cells and Cloning: Advances and Applications

8 December 2011

Number of times this article has been viewed

\author{
Olcay Y Jones' \\ Faysal Gok ${ }^{2}$ \\ Elisabeth J Rushing ${ }^{3}$ \\ Iren Horkayne-Szakaly ${ }^{4}$ \\ Atif A Ahmed ${ }^{5}$ \\ 'Department of Pediatrics, Walter \\ Reed National Military Medical \\ Center, Bethesda, MD, USA; \\ ${ }^{2}$ Department of Pediatrics, Gulhane \\ Military Medical Academy, Ankara, \\ Turkey; ${ }^{3}$ Institut für Neuropathologie, \\ Universitäts Spital Zürich, Zürich, \\ Switzerland; ${ }^{4}$ Department of \\ Neuropathology, Armed Forces \\ Institute of Pathology, Washington, \\ DC, USA; ${ }^{5}$ Department of Pathology \\ and Laboratory Medicine, Children's \\ Mercy Hospitals and Clinics, Kansas \\ City, MO, USA
}

Correspondence: Olcay Y Jones

Department of Pediatrics, Division

Pediatric Rheumatology, Walter Reed

National Military Medical Center, 890I

Wisconsin Ave, Building 19, 4th Floor,

Bethesda, MD 20889, USA

Tel + I 30I 4001619

Fax +I 30131984I3

Email olcay.jones@gmail.com

\begin{abstract}
Somatic tissue engraftment was studied in BXSB mice treated with mesenchymal stem cell transplantation. Hosts were conditioned with nonlethal radiation prior to introducing donor cells from major histocompatibility complex-matched green fluorescent protein transgenic mice. Transplant protocols differed for route of injection, ie, intravenous (i.v.) versus intraperitoneal (i.p.), and source of mesenchymal stem cells, ie, unfractionated bone marrow cells, ex vivo expanded mesenchymal stem cells, or bone chips. Tissue chimerism was determined after short (10-12 weeks) or long ( 62 weeks) posttransplant follow-up by immunohistochemistry for green fluorescent protein. Engraftment of endothelial cells was seen in several organs including liver sinusoidal cells in i.v. treated mice with ex vivo expanded mesenchymal stem cells or with unfractionated bone marrow cells. Periportal engraftment of liver hepatocytes, but not engraftment of endothelial cells, was found in mice injected i.p. with bone chips. Engraftment of adipocytes was a common denominator in both i.v. and i.p. routes and occurred during early phases post-transplant. Disease control was more robust in mice that received both i.v. bone marrow and i.p. bone chips compared to mice that received i.v. bone marrow alone. Thus, the data support potential use of mesenchymal stem cell transplant for treatment of severe lupus. Future studies are needed to optimize transplant conditions and tailor protocols that may in part be guided by fat and endothelial biomarkers. Furthermore, the role of liver chimerism in disease control and the nature of cellular communication among donor hematopoietic and mesenchymal stem cells in a chimeric host merit further investigation.
\end{abstract}

Keywords: lupus, mice, mesenchymal stem cells, endothelial cells, liver

\section{Introduction}

Mesenchymal stem cell transplantation is rapidly emerging as a treatment modality for lupus patients with unremitting disease activity. ${ }^{1,2}$ Current views emphasize the immune modulatory activities of mesenchymal stem cells and the release of potent soluble factors even without significant donor engraftment. ${ }^{3}$ Although this regimen appears efficacious, therapeutic sustainability remains unpredictable. While mesenchymal stem cell-mediated long-term disease control with tissue tropism, regeneration, and self-tolerance is the envisioned endpoint, further investigation is needed to tailor transplant protocols for lupus pathogenesis.

Preclinical studies have confirmed the complex interplay between donor mesenchymal stem cells and host tissues over time and space. For example, treatment of three prototypic lupus models with mesenchymal stem cells produced different outcomes. This is an interesting observation as lupus phenotype in all three strains derives from immune complex formation against nuclear constituents, and complement activation 
accounting for chronic inflammation and progressive renal failure. However, each strain carries unique genetic factors influencing disease immunopathogenesis for breakage of tolerance, ie, while MRL/lpr mice show dominance of T cellmediated mechanisms, New Zealand black/New Zealand white mice show B cell-mediated mechanisms, and BXSB mice show monocyte/dendritic cell-mediated mechanisms. So far, based on few preclinical reports, MRL/lpr mice respond robustly to a single intravenous (i.v.) infusion of ex vivo expanded allogeneic bone marrow mesenchymal stem cells, ${ }^{2}$ but New Zealand black/New Zealand white mice fail to improve after repeated intraperitoneal (i.p.) injections of similar cells. ${ }^{4}$ The approach in this study using BXSB mice was based on the i.p. introduction of mesenchymal stem cells in the form of bone chips. Previous experience showed that mixed chimerism following gentle conditioning induced prolonged disease remission, but only if the transplants contained both hematopoietic and mesenchymal stem cells. $^{5}$

The follow-up data on the fate of donor stem cells in vivo using paraffin-embedded tissue samples from Jones et $\mathrm{al}^{5}$ along with additional controls is presented here. The results suggest that it is possible to initiate a predictable sequence of events resulting in chimerism of the vascular bed and somatic tissues.

\section{Materials and methods}

Mixed chimerism was established as previously reported. ${ }^{5}$ Briefly, male BXSB (10 weeks of age, stock 000740; The Jackson Laboratory, Bar Harbor, ME) received nonlethal total body irradiation ( $550 \mathrm{cGy}) 4$ hours prior to transplant. Donors were $\mathrm{C} 57 \mathrm{Bl} / 6$ transgenic for green fluorescent protein (GFP) (C57BL/6-Tg[CAG-EGFP]1Osb/J; Jackson Laboratory). Bone marrow cells were harvested from long leg bones by gentle saline flush washed once and injected as unfractionated viable cell mixture in normal saline $\left(10 \times 10^{6}\right.$ per mouse). Remaining bones devoid of marrow were used to prepare bone chips; under sterile conditions four pairs of femur and four pairs of tibia were ground finely with a mortar and pestle, suspended in $4.5 \mathrm{~mL}$ saline, and injected i.p. in 0.3 cc volumes using a $20 \mathrm{~g}$ needle. Treatment arms ( $\mathrm{n}=14-20 \mathrm{mice} /$ group) included: (a) untreated BXSB mice, (b) radiation controls, (c) BMT, mice injected i.v. with unfractionated bone marrow cells $\left(1 \times 10^{7}\right.$ per mouse $)$, (d) MSC, mice injected i.p. with bone chips (equivalent to one long bone/mouse), and (e) BMT + MSC, mice transplanted both with bone marrow (i.v.) and bone chips (i.p.). Paraffin-embedded tissues from surviving mice were examined at 62 weeks. Additional groups included $550 \mathrm{cGy}$ radiated BXSB mice 10 weeks after BMT $(n=7), 6$ weeks after i.p. bone chips (ie, MSC, $\mathrm{n}=3$ ), 12 weeks after i.v. infusion of ex vivo expanded cells from cultured bone chips, which were the second passage fibroblastoid CD45- cells rapidly growing in Dulbecco's modified Eagle medium with $5 \%$ fetal calf serum $(n=3)$, and BXSB mice treated with myeloablative (950 cGy) BMT harvested 10 weeks $(\mathrm{n}=3)$ posttransplant.

Automated immunohistochemistry was performed with Leica BOND-MAX ${ }^{\mathrm{TM}}$ (Leica Microsystems GmbH, Wetzlar, Germany), and Novocastra Bond Polymer Refine Detection system (Leica Microsystems) was utilized for brown color staining with diaminobenzidine chromogen. Deparaffinized slides were rinsed and then incubated sequentially with the primary antibody, a polyclonal antiGFP antibody (cat Ab290; Abcam, Cambridge, MA), secondary antibody, and a polymer conjugate. Staining was visualized after incubation with diaminobenzidine. Tissue from GFP transgenic mice was the positive control. Tissues for negative controls were treated similarly, except for omission of the primary antibody.

\section{Results}

The following observations were made: (a) injection of i.p. mesenchymal cells led to engraftment of hepatocytes that appeared to follow engraftment of adipocytes in omentum, (b) i.v. injection of bone marrow cells or i.v. injection of ex vivo expanded mesenchymal cells resulted in engraftment of endothelial cells in multiple organs, and (c) sustained long-term disease control required cotreatment with bone marrow and bone chips.

After i.p. injection of bone chips, donor engraftment appeared to initiate in adipose tissue. This is based on the observation that mice examined 6 weeks after i.p. treatment revealed a few GFP+ cells exclusively among adipocytes harvested serendipitously along with other organs (results not shown). As shown in Figures 1 and 2, at 62 weeks posttransplant, all mice examined showed chimerism in the liver and adipose tissue, but the pattern of engraftment differed among treatment groups. In the MSC arm, engraftment of hepatocytes was periportal and appeared to correlate with the extent of tissue inflammation. However, these mice did not exhibit engraftment of the remaining organs, including kidney, lung, brain, or spleen. The BMT arm resulted in engraftment of endothelial cells in multiple organs including liver sinusoidal cells, brain choroid plexus (rich network of blood vessels covered by epithelium), as well as vascular endothelial cells in fat tissue, lung, and kidney. 


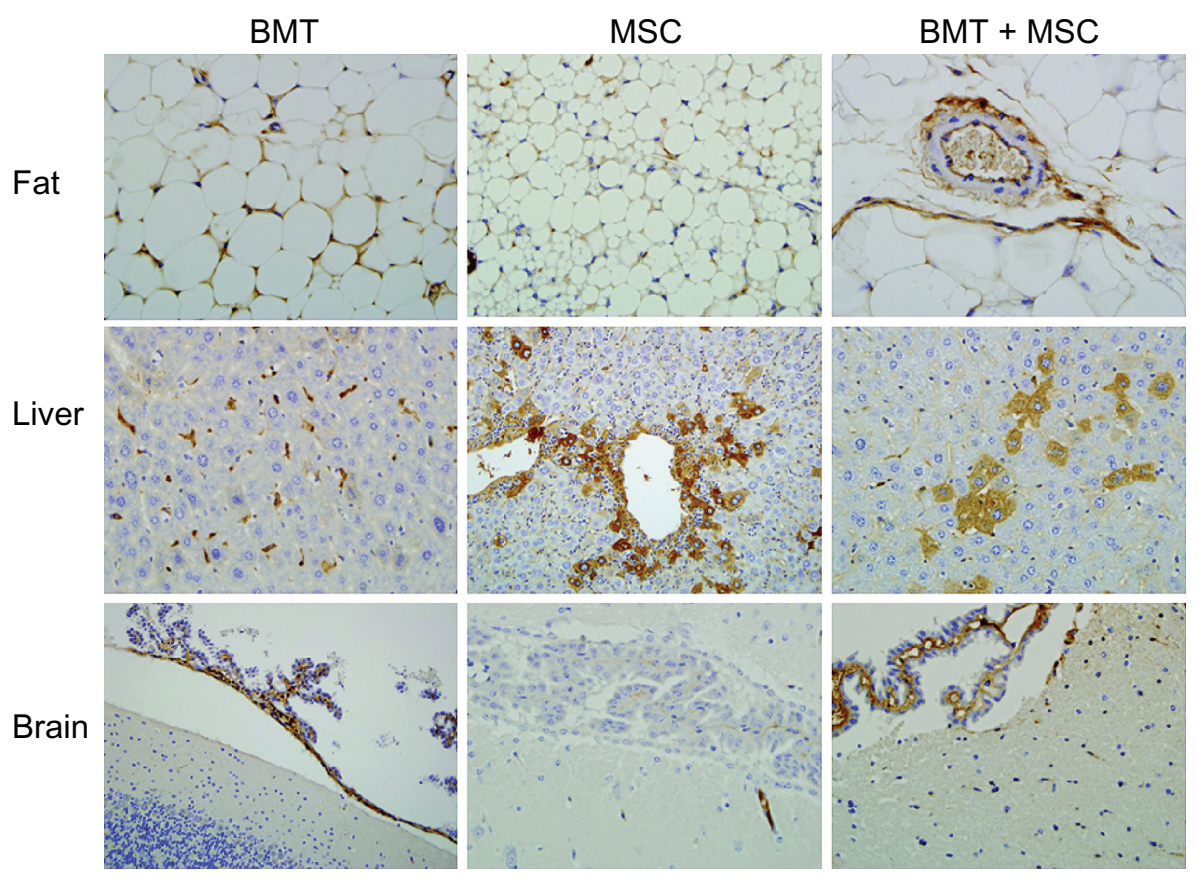

Figure I Long-term follow-up of mice treated with bone chips as source of mesenchymal stem cells injected intraperitoneally (MSC), unfractionated bone marrow cells injected intravenously (BMT), or both (MSC + BMT). Immunohistochemical staining with green fluorescent protein revealed engraftment of the vascular endothelial cells in intraabdominal fat tissue in all groups (first row), but staining of perivascular cells (most likely pericytes) was noted in BMT + MSC group. There was clear difference in the staining patterns observed in the liver tissue (second row). While BMT exhibited stained sinusoidal endothelial cells, MSC reproducibly showed chimerism among periportal hepatocytes. The mice from BMT + MSC group stained for both sinusoidal cells and hepatocytes, periportal as well as scattered parenchymal cells. The third row depicts sections of the brain tissue involving choroid plexus of the fourth ventricle (BMT-treated mouse) or choroid plexus of the inferior horn of the lateral ventricle in the region of the hippocampus (MSC- or BMT + MSC-treated mice); vascular endothelial cells of the choroid plexus along the abluminal surface and fibrovascular stroma stained positive for green fluorescent protein in BMT and BMT + MSC groups, but not the MSC group.
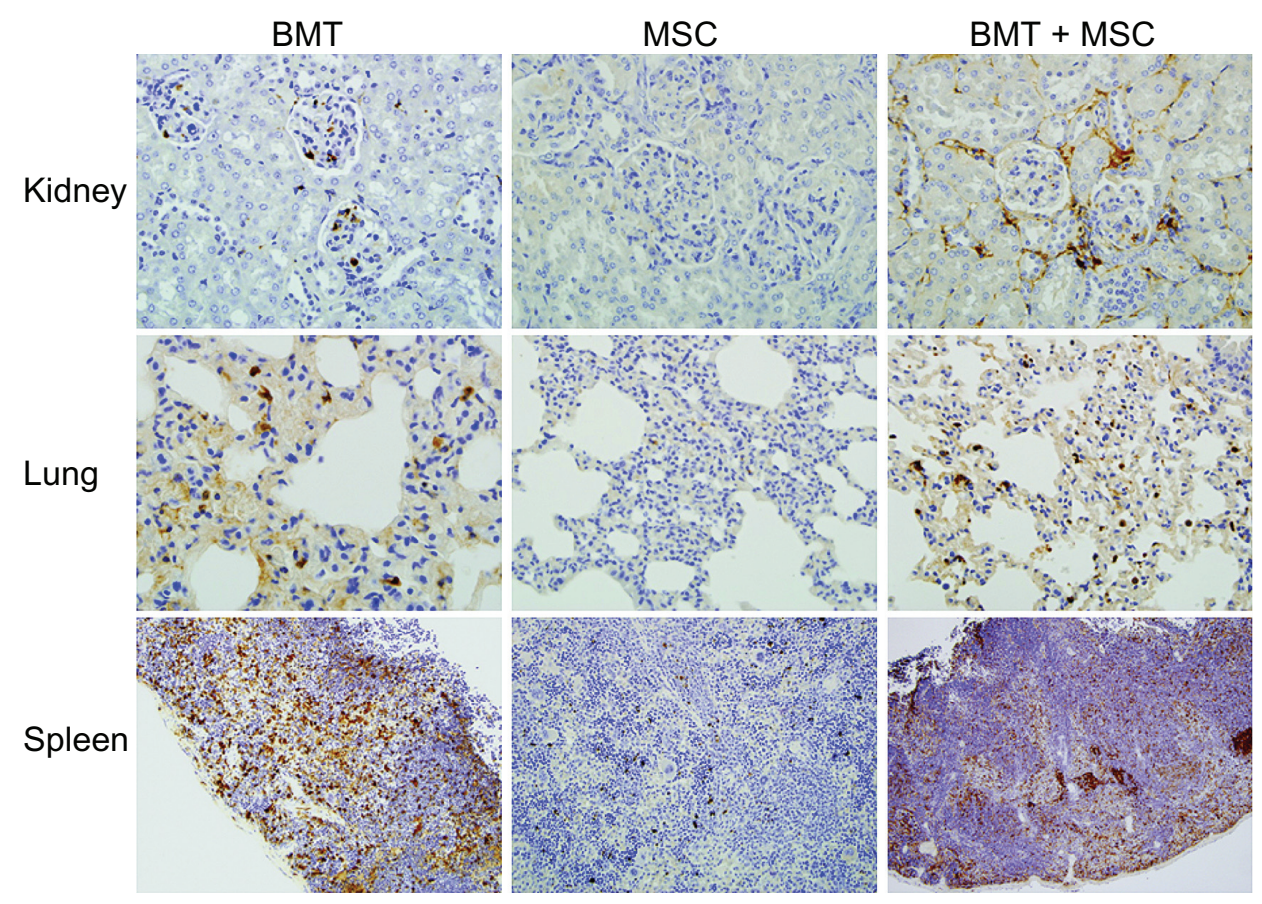

Figure 2 Long-term follow-up of mice detailed in Figure I. Donor chimerism identified by green fluorescent protein-positive cells was noted for the vascular endothelial cells in the kidney (first row) and lung (second row) parenchyma of BMT-treated mice (unfractionated bone marrow cells injected intravenously). On the other hand, MSC-treated mice (bone chips injected intraperitoneally) did not produce any vascular chimerism. The staining in the spleen (third row) corresponds to chimerism among hematopoietic cells that was similar between BMT and BMT + MSC (mice transplanted both with bone marrow and bone chips) groups, but was not noted in the MSC group. The latter finding correlated with the flow cytometry results on splenocytes. ${ }^{5}$

Abbreviations: BMT, unfractionated bone marrow cells injected intravenously; MSC, mesenchymal stem cells injected intraperitoneally. 
Focal capillary endothelial cell engraftment was seen in gut, skin, and striated muscle, but not in pancreas or brain parenchyma (data not shown).

Figure 3 summarizes results from additional treatment groups involving i.v. injection of mesenchymal stem cells in BXSB mice. When these mice were harvested 10-12 weeks posttransplant, there was clear evidence for endothelial cell engraftment in multiple organs including liver sinusoidal cells. However, there was no chimerism among hepatocytes. The pattern of engraftment was similar for the BMT arm. Furthermore, the pattern of chimerism was similar for BMT following conditioning with high (950 cGy) or low (550 cGy) dose radiation, and yielded endothelial cell engraftment similar to that in long-term follow-up group (Figures 1 and 2).

As expected, the chimerism in spleen by hematopoietic engraftment was maximal in myeloablative BMT (950 cGy) and was nondetectible in i.p. injected MSC (Figure 3 and Figure 2, respectively). Although nonmyeloablative BMT and BMT + MSC groups (Figure 2) had comparable percentages of donor hematopoietic stem cell-derived GFP+ CD45+ cells in spleen (data not shown), there were differences in the leukocyte populations. ${ }^{5}$

\section{Discussion}

The histopathologic features of chimeric BXSB following injection of donor mesenchymal stem cells (MSC arm) alone or along with bone marrow cells (BMT arm) were examined. The i.p. injection of mesenchymal stem cells was inspired by historical papers on MRL/lpr mice, where whole bone fragments were implanted under the renal capsule or subcutis to improve donor engraftment and immune competence following myeloablative $\mathrm{BMT}^{6}{ }^{6}$ Introducing donor mesenchymal stem cells via bone chips is not common practice, but based on these results appears to merit further investigation; it is a practical and inexpensive method that requires minimal ex vivo processing. In addition, this technique enables transplantation of mesenchymal stem cells in large quantities, and is suitable for introducing cells while preserving threedimensional scaffolding and natural microenvironment for optimal growth and differentiation. Furthermore, the particulate nature of bone chips may trigger inflammation that stimulates communication between donor mesenchymal stem cells and host innate immunity. However, this protocol may be more important for liver endodermal engraftment than for vascular endothelial engraftment and suggests presence of donor multipotent adult progenitor cells ${ }^{7}$ in the transplant.
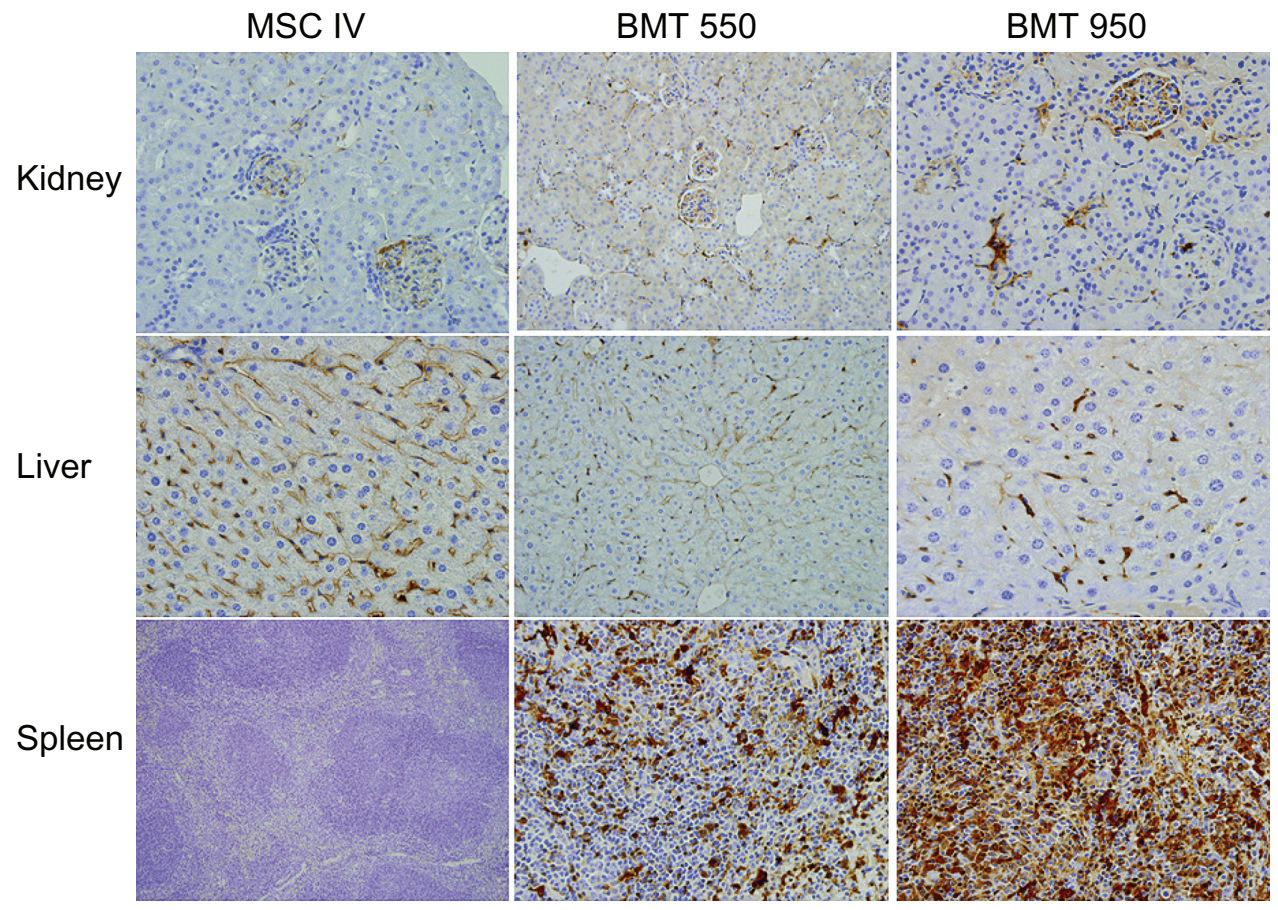

Figure 3 Short-term follow-up of mice for donor chimerism. The results of BXSB mice conditioned with low dose radiation (550 cGY) and then treated intravenously with ex vivo expanded mesenchymal stem cells from cultured bone chips are shown in the first column. The pattern of engraftment in these mice was similar to those treated with unfractionated bone marrow cells given after low dose or lethal ( $950 \mathrm{cGy}$ ) radiation. Engraftment of donor endothelial cells in the kidney and liver tissues was a common finding between these two groups regardless of the levels of hematopoietic chimerism as shown in the spleen tissues (third row).

Abbreviations: BMT, unfractionated bone marrow cells injected intravenously; MSC, mesenchymal stem cells injected intraperitoneally. 
The most prominent finding in the MSC group was the presence of donor chimerism in hepatocytes derived from endodermal stem cells. As noted, ${ }^{3}$ engraftment correlates with inflammatory changes in the liver, and was prominent in mice with active lupus. Adipocytes derived from mesodermal stem cells were the first to differentiate from i.p. bone chips. This may reflect the recent observation that vascular omentum is a suitable stem cell niche ${ }^{8}$ and that the inflammatory milieu can facilitate liver regeneration. ${ }^{9}$ Adipocytes are known potent sources of nutrition and vascular endothelial growth factor and share a common progenitor with endothelial cells. ${ }^{10}$ In the current study, there were a few capillary structures with GFP+ endothelial cells found in the vicinity of adipocytes, even in the absence of cotransplanted bone marrow. The topology of engraftment suggests the importance of portal circulation for the homing of stem cells to liver and that GFP+ pericytes may play a role in hematologic dissemination of progenitors. ${ }^{11}$ While the impact of donor hepatocytes in disease activity is unknown, it may encompass immunomodulation and systemic influences on tissue tropism and regeneration by soluble factors such as vascular endothelial growth factor. Importantly, for cell-based treatments of hepatic tissue, ${ }^{12}$ further studies are warranted to explore the use of i.p. bone chips for other therapeutic applications, including chronic liver diseases.

Immune complex mediated vasculopathy and tissue damage are hallmarks of lupus pathogenesis. Endothelial engraftment is expected to be crucial for long-term disease control. ${ }^{13}$ The results of the current study provide clear evidence that this goal is attainable using cell-based treatment modalities. Multisystem engraftment of endothelial cells was found if progenitor cells were introduced by i.v. route, but not i.p. route. Although further studies are warranted, bone chips appear to harbor not only the bone marrow stem cell-derived endothelial cells, as seen in i.v. injection of mesenchymal stem cells (Figure 3), but also adipose stem cell-derived endothelial cells, as seen in MSC arm (Figure 1). Thus, the route of treatment determines the extent of endothelial cell chimerism. Furthermore, organ specific factors may influence the patterns observed; ie, presence of GFP+ sinusoidal cells in the liver and choroid plexus in the brain.

In the current model, it was documented that both MSC and BMT are necessary to achieve sustained disease control with a nonmyeloablative regimen. Alternatively, previous reports found that myeloablative transplants with BMT alone were able to curtail disease activity. ${ }^{14}$ Thus, disease control can be achieved either through ablation of immune mediated tissue damage, ie, by replacing host immune system, or by establishing a "steady state" where long-term modulation of the immune system can be achieved by donor cells. An earlier study suggested that sustained disease control can be accomplished in New Zealand black/New Zealand white mice after nonmyeloablative transplant of purified hematopoietic stem cells. ${ }^{15}$ Accordingly, it is tempting to speculate that BXSB might have additional strain specific dependence on donor somatic cells to down regulate tissue factors involved in antigenic stimulation and inflammatory cascades, and/or for tissue repair and regeneration.

\section{Acknowledgments}

We thank Dr Joe M Jones for critical review of data and manuscript. The views expressed in this article are those of the authors and do not necessarily reflect the official policy or position of the Department of Defense, nor the US Government. This work was supported by the Eleanor Naylor Dana Charitable Trust and Children's Research Institute, Washington, DC.

\section{Disclosure}

The authors report no conflicts of interest in this work.

\section{References}

1. Zhang $\mathrm{H}$, Zeng $\mathrm{X}$, Sun L. Allogenic bone-marrow derived mesenchymal stem cells transplantation as a novel therapy for systemic lupus erythematosus. Expert Opin Biol Ther. 2010;10(5): 701-709.

2. Sun L, Akiyama K, Zhang H, et al. Mesenchymal stem cell transplantation reverses multiorgan dysfunction in systemic lupus erythematosus mice and humans. Stem Cells. 2009;27(6):1421-1432.

3. Caplan AI. What's in a name? Tissue Eng Part A. 2010;16(8): 2415-2417.

4. Youd M, Blickarz C, Woodworth L, et al. Allogeneic mesenchymal cells do not protect NZB $\times$ NZW F1 mice from developing lupus disease. Clin Exp Immunol. 2010;161(1):176-186.

5. Jones OY, Lacson A, Zeng X, et al. Long-term follow-up after nonmyeloablative transplant of bone and marrow in BXSB mice. Lupus. 2009;18(9):813-821.

6. Ishida $\mathrm{T}$, Inaba $\mathrm{M}$, Hisha $\mathrm{H}$, et al. Requirement of donor-derived stromal cells in the bone marrow for successful allogeneic bone marrow transplantation. Complete prevention of recurrence of autoimmune diseases in MRL/MP-lpr/lpr mice by transplantation of bone marrow plus bones (stromal cells) from the same donor. J Immunol. 1994;152(6):3119-3127.

7. Schwartz RE, Reyes M, Koodie L, et al. Multipotent adult progenitor cells from bone marrow differentiate into functional hepatocyte-like cells. J Clin Invest. 2002;109(10):1291-1302.

8. Litbarg NO, Gudehithlu KP, Sethupathi P, Arruda JA, Dunea G, Singh AK. Activated omentum becomes rich in factors that promote healing and tissue regeneration. Cell Tissue Res. 2007;328(3):487-497.

9. Singh AK, Pancholi N, Patel J, et al. Omentum facilitates liver regeneration. World J Gastroenterol. 2009;15(9):1057-1064. 
10. Planat-Benard V, Silvestre JS, Cousin B, et al. Plasticity of human adipose lineage cells toward endothelial cells: physiological and therapeutic perspectives. Circulation. 2004;109(5):656-663.

11. Corselli M, Chen CW, Crisan M, Lazzari L, Peault B. Perivascular ancestors of adult multipotent stem cells. Arterioscler Thromb Vasc Biol. 2010;30(6):1104-1109.

12. Sancho-Bru P, Najimi M, Caruso M, et al. Stem and progenitor cells for liver repopulation: can we standardise the process from bench to bedside? Gut. 2009;58(4):594-603.

13. Distler JH, Beyer C, Schett G, Luscher TF, Gay S, Distler O. Endothelial progenitor cells novel players in the pathogenesis of rheumatic diseases. Arthritis Rheum. 2009;60(11):3168-3179.
14. Ikehara S, Good RA, Nakamura T, et al. Rationale for bone marrow transplantation in the treatment of autoimmune diseases. Proc Natl Acad Sci U S A. 1985;82(8):2483-2487.

15. Smith-Berdan S, Gille D, Weissman IL, Christensen JL. Reversal of autoimmune disease in lupus-prone New Zealand black/New Zealand white mice by nonmyeloablative transplantation of purified allogeneic hematopoietic stem cells. Blood. 2007;110(4):1370-1378.

\section{Publish your work in this journal}

Stem Cells and Cloning: Advances and Applications is an international, peer-reviewed, open access journal. Areas of interest in stem cell research include: Embryonic stem cells; Adult stem cells; Blastocysts; Cordblood stem cells; Stem cell transformation and culture; Therapeutic cloning; Umbilical cord blood and bone marrow cells; Laboratory, animal and human therapeutic studies; Philosophical and ethical issues related to stem cell research. This journal is indexed on CAS. The manuscript management system is completely online and includes a quick and fair peer-review system. Visit http://www.dovepress.com/ testimonials.php to read real quotes from published authors.

Submit your manuscript here: http://www.dovepress.com/stem-cells-and-cloning-advances-and-applications-journal 